\section{Mortalidade por COVID-19 padronizada por idade nas capitais das diferentes regiões do Brasil}

\author{
Age-adjusted COVID-19 mortality in state capitals \\ in different regions of Brazil
}

\author{
Mortalidad por COVID-19 estandarizada por \\ edad en las capitales de las diferentes \\ regiones de Brasil
}

COMUNICAÇÃO BREVE

BRIEF COMMUNICATION
Gulnar Azevedo e Silva 1
Beatriz Cordeiro Jardim 2
Paulo Andrade Lotufo 3

doi: 10.1590/0102-311X00039221

\section{Resumo}

O crescimento acentuado de casos e óbitos por COVID-19 tem levado à grande sobrecarga do sistema de saúde no Brasil, em especial em cidades como Manaus (Amazonas), Rio de Janeiro e São Paulo. A descrição do impacto da pandemia tem se baseado em números absolutos ou taxas de mortalidade brutas, não considerando o padrão de distribuição das faixas etárias nas diferentes regiões do país. Este estudo tem por objetivo comparar as taxas de mortalidade brutas por COVID-19 com as taxas padronizadas por idade nas capitais dos estados brasileiros e no Distrito Federal. As informações sobre óbito foram acessadas no Sistema de Informação de Vigilância da Gripe (SIVEP-Gripe), e os denominadores populacionais foram baseados nas estimativas disponibilizadas pelo Ministério da Saúde. Para o cálculo das taxas padronizadas por idade, utilizou-se a estrutura etária da população do Brasil estimada para 2020. Os resultados mostram que as maiores taxas brutas foram em $\mathrm{Ma}$ naus (253,6/100 mil) e no Rio de Janeiro (253,2/100 mil). Após padronização por idade, houve aumento expressivo das taxas na Região Norte. A maior taxa ajustada foi vista em Manaus (412,5/100 mil) onde 33\% de óbitos por COVID-19 ocorreram entre menores de 60 anos. A mortalidade em Manaus acima de 70 anos foi o dobro se comparada à do Rio de Janeiro e o triplo se comparada à de São Paulo. A utilização de taxas de mortalidade padronizadas por idade elimina vieses interpretativos, expondo, de forma marcante, o peso ainda maior da COVID-19 na Região Norte do país.

COVID-19; Desigualdades em Saúde; Mortalidade

\author{
Correspondência \\ G. Azevedo e Silva \\ Instituto de Medicina Social, Universidade do Estado do Rio de \\ Janeiro. \\ Rua São Francisco Xavier 524, 7o andar, Blocos D e E, Rio de \\ Janeiro, RJ 20550-900, Brasil. \\ gulnar@ims.uerj.br \\ ${ }^{1}$ Instituto de Medicina Social, Universidade do Estado do Rio de \\ Janeiro, Rio de Janeiro, Brasil. \\ 2 Instituto Nacional de Câncer José Alencar Gomes da Silva, Rio \\ de Janeiro, Brasil. \\ 3 Hospital Universitário, Universidade de São Paulo, São Paulo, \\ Brasil.
}




\section{Introdução}

A pandemia de COVID-19 teve o primeiro caso confirmado no país em 25 de fevereiro de 2020, e as análises filogenéticas das cepas virais isoladas no país confirmam que houve entrada massiva por São Paulo, Rio de Janeiro e, também, em Fortaleza (Ceará) 1. Desde então, descreveu-se aumento expressivo na mortalidade geral e excesso de mortes em 2020, comparado aos anos anteriores 2,3. Ao fim de 2020, foi possível contabilizar 24\% a mais de mortes em 2020 em relação ao quinquênio 20152019, variando entre 40\% na Região Norte e 10\% na Região Sul (Conselho Nacional de Secretários de Saúde. Painel CONASS - COVID-19. http://www.conass.org.br/painelconasscovid19/, acessado em 07/Fev/2021).

O crescimento acentuado de casos e óbitos levou a que algumas cidades, como Rio de Janeiro, São Paulo e Manaus (Amazonas), chegassem a condições extremas que resultaram em grande sobrecarga do sistema de saúde, ocasionando altos índices de morte hospitalar ${ }^{4}$. A descrição e o impacto da pandemia, contudo, têm se baseado em números absolutos ou taxas de mortalidade brutas 5 , o que não leva em conta o padrão de distribuição das faixas etárias nas diferentes regiões do país.

$\mathrm{O}$ objetivo deste estudo foi estimar a diferença entre as taxas de mortalidade brutas e as taxas padronizadas por idade por COVID-19 nas capitais dos estados brasileiros e no Distrito Federal.

\section{Métodos}

\section{Fonte de dados}

Inicialmente, foram comparadas as informações geradas em três sistemas diferentes que disponibilizam dados atualizados sobre o total de óbitos registrados por COVID-19 no Brasil: Sistema de Informação de Vigilância da Gripe - SIVEP-Gripe (Departamento de Informática do SUS. https:// opendatasus.saude.gov.br, acessado em 07/Fev/2021); MonitoraCOVID-19 do Instituto de Comunicação e Informação Científica e Tecnológica em Saúde, Fundação Oswaldo Cruz (https://bigdatacovid19.icict.fiocruz.br/, acessado em 07/Fev/2021) e; o Sistema de Registro Civil, disponibilizado pela Associação Nacional dos Registradores de Pessoas Naturais - ARPEN (Portal da Transparência. Registro civil. https://transparencia.registrocivil.org.br, acessado em 31/Jan/2021).

Embora as coberturas das informações sejam bem próximas para os municípios do Rio de Janeiro e de São Paulo, os dados do Sistema de Registro Civil mostram uma proporção inferior de óbitos para Manaus (Figura 1). Optou-se, assim, por utilizar os dados registrados no SIVEP-Gripe de óbitos por COVID-19 por idade nas capitais dos estados e Distrito Federal até a data de 30 de janeiro de 2021, disponíveis em 1o de fevereiro de 2021. Os denominadores populacionais para o ano de 2020 para o Brasil e municípios de residência referentes às capitais dos estados e do Distrito Federal por faixa etária foram os apresentados nas estimativas preliminares disponibilizadas pelo Ministério da Saúde (Departamento de Informática do SUS. População residente: estudo de estimativas populacionais por município, idade e sexo 2000-2020 - Brasil. http://tabnet.datasus.gov.br/cgi/deftohtm.exe?popsvs/ cnv/popbr.def, acessado em 08/Fev/2021).

\section{Cálculo das taxas}

Foram calculadas as taxas brutas de mortalidade por COVID-19 por 100 mil habitantes para todas as capitais e Distrito Federal com os dados acumulados entre os meses de março de 2020 até 30 de janeiro de 2021. Em seguida, foram calculadas as taxas padronizadas por idade (com intervalos de 10 em 10 anos) pelo método direto 6 , tendo, como referência, a estrutura etária da população do Brasil estimada para o ano de 2020 (Departamento de Informática do SUS. População residente: estudo de estimativas populacionais por município, idade e sexo 2000-2020 - Brasil. http://tabnet.datasus.gov. $\mathrm{br} / \mathrm{cgi} / \mathrm{deftohtm}$.exe?popsvs/cnv/popbr.def, acessado em 08/Fev/2021). 


\section{Figura 1}

Mortes acumuladas por COVID-19 em Manaus (Amazonas), Rio de Janeiro e São Paulo, Brasil, disponibilizadas pelo MonitoraCOVID-19, SIVEP-Gripe e Sistema de Registro Civil entre os meses de dezembro de 2020 e janeiro de 2021.

1a) Manaus

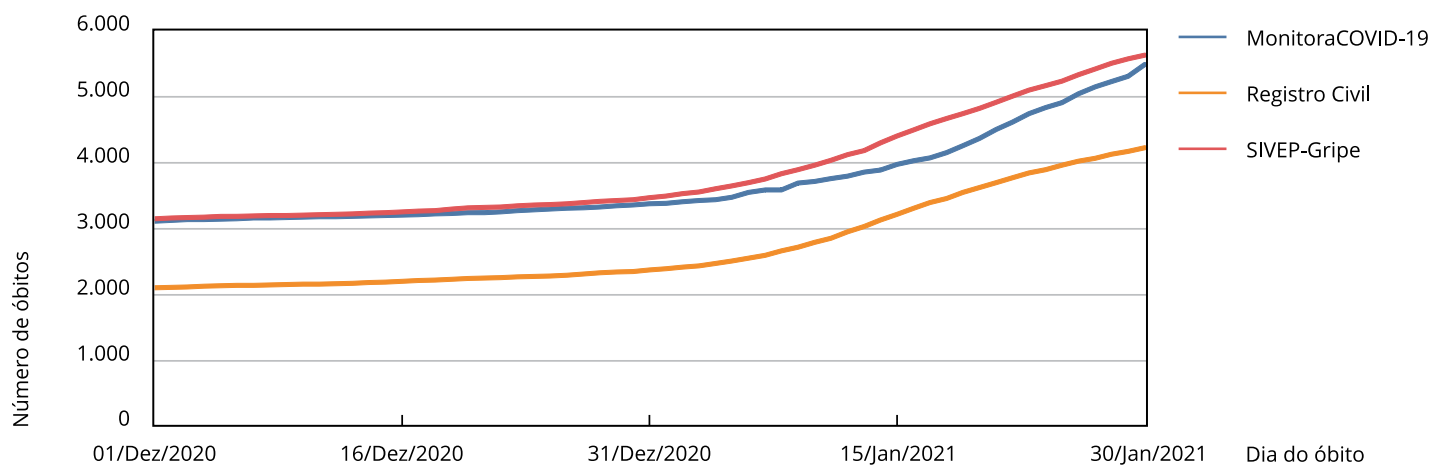

1b) Rio de Janeiro

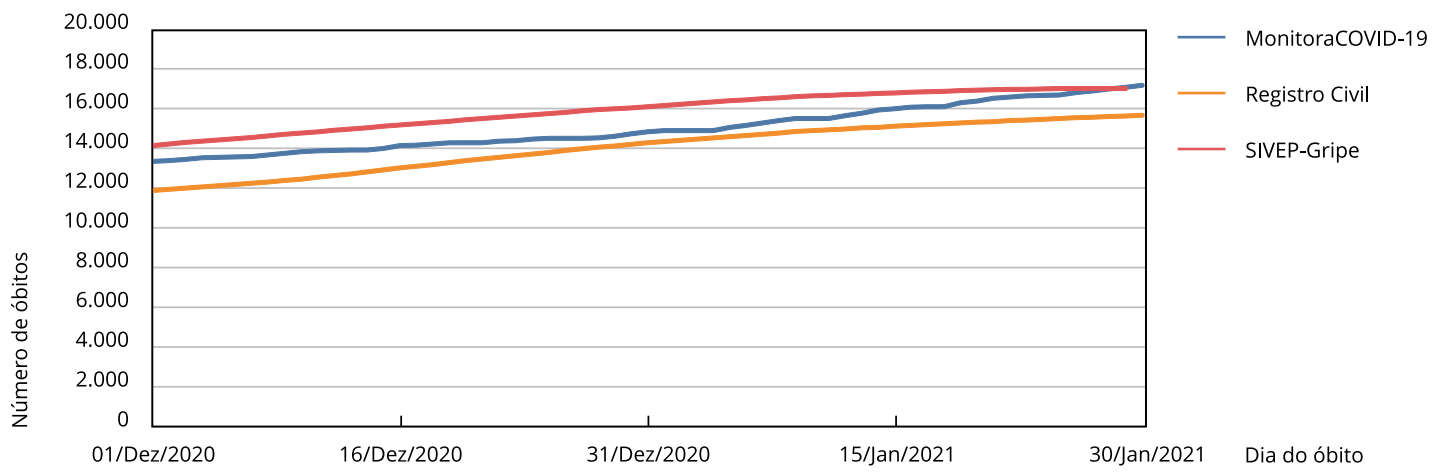

1c) São Paulo

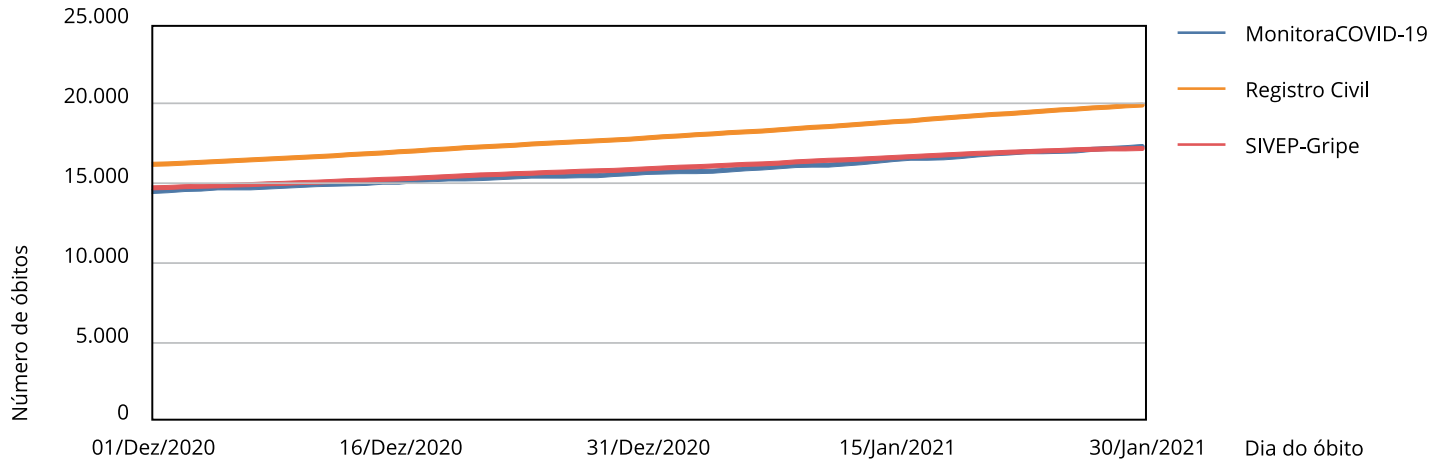

Fontes: MonitoraCOVID-19 (Instituto de Comunicação e Informação Científica e Tecnológica em Saúde, Fundação Oswaldo Cruz. https://bigdata-covid19. icict.fiocruz.br/, acessado em 07/Fev/2021); Sistema de Informação de Vigilância da Gripe - SIVEP-Gripe (Departamento de Informática do SUS. https://opendatasus.saude.gov.br, acessado em 07/Fev/2021); e Sistema de Registro Civil (Associação Nacional dos Registradores de Pessoas Naturais. Portal da Transparência. Registro civil. https://transparencia.registrocivil.org.br, acessado em 31/Jan/2021). 
Considerando que as taxas seguem uma distribuição de Poisson e que o número de óbitos é consideravelmente alto na população de estudo, foi utilizada uma aproximação normal para calcular os intervalos de confiança para as diferenças de taxas de mortalidade pela equação:

$$
I C 95 \%=\left(\left(\frac{E-O}{N}\right) \pm 1,96 \times \sqrt{\frac{E+O}{N^{2}}}\right) \times 10^{5}
$$

Onde $E$ é o número de óbitos esperados a partir da taxa padronizada, $O$ é o número de óbitos ocorridos, e $N$ é o número de habitantes na capital.

As taxas brutas e padronizadas foram consideradas estatisticamente diferentes quando o intervalo de 95\% de confiança (IC95\%) para a diferença não continha o zero.

Todos os cálculos foram realizados no programa estatístico Stata (https://www.stata.com).

\section{Resultados}

As maiores taxas brutas de mortalidade foram observadas em Manaus (253,6/100 mil) e no Rio de Janeiro (253,2/100 mil), enquanto Palmas (Tocantins) e Florianópolis (Santa Catarina) tiveram as mais baixas (70,5 e 79,4 por 100 mil, respectivamente) (Tabela 1$)$.

Com a padronização por idade, a mais alta taxa de mortalidade foi observada em Manaus (412,5/100 mil habitantes), e a mais baixa, em Florianópolis (67,2/100 mil habitantes). Em todas as capitais da Região Norte, houve aumento na taxa padronizada por idade. Os maiores incrementos foram observados nas capitais da Região Norte (98,12\% em Boa Vista - Roraima - e 92,57\% em Palmas).

$\mathrm{Na}$ Região Nordeste, os maiores aumentos nas taxas padronizadas por idade foram observados em Teresina - Piauí (25,23\%) e São Luís - Maranhão (25,09\%). As diferenças estatisticamente significativas foram observadas também em Fortaleza, Maceió (Alagoas) e Aracaju (Sergipe). Em João Pessoa (Paraíba) e Natal (Rio Grande do Norte), não houve diferença entre as taxas, e, apenas, em Recife (Pernambuco), foi constatada queda. Na Região Centro-oeste, houve aumento nas taxas em Brasília, Cuiabá (Mato Grosso) e Goiânia (Goiás).

Em sentido inverso, em todas as capitais das regiões Sul e Sudeste, as taxas padronizadas por idade foram inferiores às taxas brutas.

Nota-se, em Manaus, uma diferença por faixas etárias muito distinta em relação a São Paulo e Rio de Janeiro. A proporção de habitantes maiores de 60 anos é de 8,4\% em Manaus, 16,1\% em São Paulo e 18,9\% no Rio de Janeiro. Em contraste, a proporção de óbitos pela COVID-19 em menores de 60 anos em Manaus foi de 33\%, e, no Rio de Janeiro e São Paulo, 22\%. É marcante o peso da mortalidade nas faixas etárias de 70-79 e 80 anos e mais: as taxas de Manaus dobram se comparadas às do Rio de Janeiro e triplicam em relação às de São Paulo (Tabela 2).

\section{Discussão}

Após o ajuste por idade, as taxas padronizadas nas capitais da Região Norte ficaram expressivamente maiores, com destaque para a cidade de Manaus, onde o risco de morte pela COVID-19 foi o dobro em relação ao Rio de Janeiro e o tripo a São Paulo, onde ocorreram os primeiros casos.

A mortalidade verificada em Manaus e outros municípios da região Amazônica, muito anunciada na imprensa leiga e documentada na literatura científica ${ }^{7}$, só não foi ainda maior porque a estrutura etária da população é jovem se comparada a outras cidades, como São Paulo e Rio de Janeiro que têm mais do dobro de habitantes com mais de 60 anos de idade. No entanto, a mortalidade precoce em Manaus foi substancialmente maior do que em outras capitais como São Paulo e Rio de Janeiro.

Deve ser considerado, contudo, que taxas de mortalidade, sejam brutas ou padronizadas por idade, não são a melhor medida de óbito em uma epidemia com temporalidade distinta em um território extenso como o do Brasil. No entanto, desde o segundo semestre de 2020, a epidemia da COVID-19 já tinha atingido todo o território nacional, o que permite comparações, ao menos, entre as capitais dos estados. 
Tabela 1

Número de óbitos acumulados, população residente, taxas de mortalidade por COVID-19 brutas e padronizadas por idade nas capitais dos 26 estados brasileiros e do Distrito Federal.

\begin{tabular}{|c|c|c|c|c|c|c|}
\hline Região/Capital & Óbitos & População & Taxa bruta * & Taxa padronizada ** & Diferença (IC95\%) * & Variação (\%) \\
\hline \multicolumn{7}{|l|}{ Norte } \\
\hline Belém & 2.525 & 1.499 .641 & 168,37 & 181,61 & $13,24(3,77 ; 22,71)$ & 7,86 \\
\hline Boa Vista & 522 & 419.652 & 124,39 & 246,44 & $122,05(103,62 ; 140,47)$ & 98,12 \\
\hline Macapá & 533 & 512.902 & 103,92 & 189,77 & $85,85(71,02 ; 100,68)$ & 82,61 \\
\hline Manaus & 5.629 & 2.219 .580 & 253,61 & 412,54 & $158,94(148,20 ; 169,67)$ & 62,67 \\
\hline Palmas & 216 & 306.296 & 70,52 & 135,80 & $65,28(49,19 ; 81,36)$ & 92,57 \\
\hline Porto Velho & 933 & 539.354 & 172,98 & 304,76 & $131,77(113,33 ; 150,22)$ & 76,18 \\
\hline Rio Branco & 512 & 413.418 & 123,85 & 195,97 & $72,13(54,89 ; 89,36)$ & 58,23 \\
\hline \multicolumn{7}{|l|}{ Nordeste } \\
\hline Aracaju & 998 & 664.908 & 150,10 & 173,12 & $23,03(9,36 ; 36,69)$ & 15,34 \\
\hline Fortaleza & 4.691 & 2.686 .612 & 174,61 & 191,75 & $17,15(9,91 ; 24,38)$ & 9,82 \\
\hline João Pessoa & 1.259 & 817.511 & 154,00 & 163,31 & $9,31(-2,91 ; 21,52)$ & 6,05 \\
\hline Maceió & 1.229 & 1.025 .360 & 119,86 & 140,93 & $21,07(11,18 ; 30,95)$ & 17,58 \\
\hline Natal & 1.209 & 890.480 & 135,77 & 135,24 & $-0,53(-11,34 ; 10,28)$ & $-0,39$ \\
\hline Recife & 3.352 & 1.653 .461 & 202,73 & 184,54 & $-18,18(-27,67 ;-8,70)$ & $-8,97$ \\
\hline Salvador & 3.347 & 2.886 .698 & 115,95 & 121,84 & $5,90(0,27 ; 11,52)$ & 5,08 \\
\hline São Luís & 1.142 & 1.108 .975 & 102,98 & 128,81 & $25,83(16,87 ; 34,79)$ & 25,08 \\
\hline Teresina & 1.254 & 868.075 & 144,46 & 180,90 & $36,44(24,44 ; 48,44)$ & 25,22 \\
\hline \multicolumn{7}{|l|}{ Sudeste } \\
\hline Belo Horizonte & 2.241 & 2.521 .564 & 88,87 & 70,23 & $-18,65(-23,57 ;-13,72)$ & $-20,97$ \\
\hline Rio de Janeiro & 17.084 & 6.747 .815 & 253,18 & 195,74 & $-57,44(-62,49 ;-52,38)$ & $-22,69$ \\
\hline São Paulo & 17.346 & 12.325 .232 & 140,74 & 125,35 & $-15,38(-18,26 ;-12,50)$ & $-10,94$ \\
\hline Vitória & 637 & 365.855 & 174,11 & 145,37 & $-28,74(-47,06 ;-10,43)$ & $-16,51$ \\
\hline \multicolumn{7}{|l|}{ Sul } \\
\hline Curitiba & 2.560 & 1.948 .626 & 131,37 & 117,42 & $-13,95(-20,96 ;-6,95)$ & $-10,62$ \\
\hline Florianópolis & 404 & 508.826 & 79,40 & 67,22 & $-12,17(-22,70 ;-1,65)$ & $-15,34$ \\
\hline Porto Alegre & 2.129 & 1.488 .252 & 143,05 & 101,04 & $-42,02(-49,95 ;-34,08)$ & $-29,37$ \\
\hline \multicolumn{7}{|l|}{ Centro-oeste } \\
\hline Brasília & 4.324 & 3.055 .149 & 141,53 & 177,11 & $35,58(29,25 ; 41,91)$ & 25,14 \\
\hline Campo Grande & 1.294 & 906.092 & 142,81 & 144,67 & $1,86(-9,18 ; 12,90)$ & 1,30 \\
\hline Cuiabá & 624 & 617.848 & 101,00 & 118,97 & $17,98(6,28 ; 29,67)$ & 17,79 \\
\hline Goiânia & 2.295 & 1.536 .097 & 149,40 & 160,28 & $10,87(2,07 ; 19,67)$ & 7,28 \\
\hline
\end{tabular}

IC95: intervalo de 95\% de confiança.

* Por 100 mil habitantes;

** Por 100 mil habitantes e padronizada por idade pela população Brasil: estimativa 2020 (Departamento de Informática do SUS. População residente: estudo de estimativas populacionais por município, idade e sexo 2000-2020 - Brasil. http://tabnet.datasus.gov.br/cgi/deftohtm.exe?popsvs/cnv/popbr. def, acessado em 08/Fev/2021).

Mudanças em curso no padrão de mortalidade, natalidade e fecundidade no país não se dão de forma uniforme e simultânea, mas têm sido determinantes para moldar as desigualdades entre as regiões 8 . Nas regiões Norte e Nordeste, tanto a mortalidade quanto a fertilidade são maiores, e a estrutura etária, mais jovem 9 .

Embora os indicadores de saúde tenham melhorado substancialmente no país entre 1990 e 2016, a carga de doença ainda permanece muito alta nos estados das regiões Norte e Nordeste se comparada à das regiões Sudeste e Sul 10. Essa condição se repetiu e se intensificou com a COVID-19, em especial na Região Norte. 
Tabela 2

Número de óbitos observados, população residente, taxa de mortalidade específica por faixa etária e número de óbitos esperados após padronização em Manaus (Amazonas), Rio de Janeiro e São Paulo, Brasil.

\begin{tabular}{|c|c|c|c|c|c|}
\hline \multirow[t]{2}{*}{ Cidade/Idade (anos) } & \multirow{2}{*}{$\begin{array}{c}\text { Óbitos } \\
\text { observados }\end{array}$} & \multicolumn{2}{|c|}{ População } & \multirow[t]{2}{*}{ Taxa específica * } & \multirow{2}{*}{ Óbitos esperados ** } \\
\hline & & $\mathbf{n}$ & $\%$ & & \\
\hline \multicolumn{6}{|l|}{ Manaus } \\
\hline $0-9$ & 25 & 355.277 & 16,01 & 7,04 & 21,67 \\
\hline $10-19$ & 19 & 395.462 & 17,82 & 4,81 & 15,41 \\
\hline $20-29$ & 74 & 405.722 & 18,28 & 18,24 & 65,42 \\
\hline $30-39$ & 245 & 377.118 & 16,99 & 64,97 & 233,11 \\
\hline $40-49$ & 546 & 303.661 & 13,68 & 179,81 & 551,37 \\
\hline $50-59$ & 889 & 197.099 & 8,88 & 451,04 & $1.128,75$ \\
\hline $60-69$ & 1.457 & 115.258 & 5,19 & $1.264,12$ & $2.217,16$ \\
\hline $70-79$ & 1.342 & 49.724 & 2,24 & $2.698,90$ & $2.552,55$ \\
\hline $80+$ & 1.032 & 20.259 & 0,91 & $5.094,03$ & $2.371,28$ \\
\hline Total & 5.629 & 2.219 .580 & 100,00 & & $9.156,73$ \\
\hline Taxa bruta * & 253,61 & & & & \\
\hline Taxa padronizada $*, \star \star$ & 412,54 & & & & \\
\hline \multicolumn{6}{|l|}{ Rio de Janeiro } \\
\hline $0-9$ & 30 & 806.837 & 11,96 & 3,72 & 34,81 \\
\hline $10-19$ & 28 & 835.964 & 12,39 & 3,35 & 32,66 \\
\hline $20-29$ & 153 & 987.608 & 14,64 & 15,49 & 168,93 \\
\hline $30-39$ & 458 & 1.054 .322 & 15,62 & 43,44 & 473,86 \\
\hline $40-49$ & 1.038 & 966.380 & 14,32 & 107,41 & $1.001,35$ \\
\hline $50-59$ & 1.982 & 819.557 & 12,15 & 241,84 & $1.839,92$ \\
\hline $60-69$ & 3.822 & 673.903 & 9,99 & 567,14 & $3.024,09$ \\
\hline $70-79$ & 4.451 & 382.978 & 5,68 & $1.162,21$ & $3.341,68$ \\
\hline $80+$ & 5.122 & 220.266 & 3,26 & $2.325,37$ & $3.290,84$ \\
\hline Total & 17.084 & 6.747 .815 & 100,00 & & $13.208,13$ \\
\hline Taxa bruta * & 253,18 & & & & \\
\hline Taxa padronizada *,** & 195,74 & & & & \\
\hline \multicolumn{6}{|l|}{ São Paulo } \\
\hline $0-9$ & 15 & 1.569 .846 & 12,93 & 0,96 & 16,34 \\
\hline $10-19$ & 28 & 1.641 .743 & 13,52 & 1,71 & 30,37 \\
\hline $20-29$ & 130 & 1.827 .397 & 15,05 & 7,11 & 141,69 \\
\hline $30-39$ & 491 & 2.044 .130 & 16,83 & 24,02 & 478,59 \\
\hline $40-49$ & 992 & 1.815 .252 & 14,95 & 54,65 & 930,56 \\
\hline $50-59$ & 2.100 & 1.443 .720 & 11,89 & 145,46 & $2.021,35$ \\
\hline $60-69$ & 3.846 & 1.083 .124 & 8,92 & 355,08 & $3.458,31$ \\
\hline $70-79$ & 4.470 & 589.507 & 4,85 & 758,26 & $3.982,28$ \\
\hline $80+$ & 5.274 & 310.513 & 2,56 & $1.698,48$ & $4.390,42$ \\
\hline Total & 17.346 & 12.325 .232 & 100,00 & & $15.449,91$ \\
\hline Taxa bruta * & 140,74 & & & & \\
\hline Taxa padronizada *,** & 125,35 & & & & \\
\hline
\end{tabular}

* Por 100 mil habitantes;

** Calculado com base na estrutura de idade da população Brasil: estimativa 2020 (Departamento de Informática do SUS. População residente: estudo de estimativas populacionais por município, idade e sexo 2000-2020 - Brasil. http://tabnet.datasus.gov.br/cgi/deftohtm.exe?popsvs/cnv/popbr.def, acessado em 08/Fev/2021). 
A limitação principal deste estudo é decorrente do processo dinâmico da contabilização de mortes pela COVID-19 que ocorre em todos os países. No entanto, o SIVEP-Gripe é um sistema que tem se mostrado relativamente homogêneo na captura e divulgação de dados entre as capitais brasileiras. Os nossos dados estão em consonância com outras fontes como a do Conselho Nacional de Secretários de Saúde (CONASS) que já vinha chamando atenção sobre o excesso de mortes verificado na Região Norte (Painel CONASS - COVID-19. http://www.conass.org.br/painelconasscovid19/, acessado em 07/Fev/2021).

Por fim, é importante levar em conta que outros fatores, além da estrutura etária, podem ser decisivos para aumentar o risco de morte independentemente da idade. A ausência de políticas preventivas adequadas e a baixa capacidade de resolutividade da rede assistencial expõem um contexto de grande desigualdade socioeconômica e iniquidade de acesso aos serviços de saúde 11 .

Conclui-se que a comparação de taxas de mortalidade padronizadas por idade elimina vieses interpretativos expondo, de forma marcante, o peso ainda maior da COVID-19 na Região Norte do Brasil.

\section{Colaboradores}

G. Azevedo e Silva contribuiu com a concepção e desenho do estudo, análise dos dados e interpretação dos resultados, redação do manuscrito e aprovação da versão final. B. C. Jardim colaborou com a análise dos dados, interpretação dos resultados e aprovação da versão final do manuscrito. P. A. Lotufo contribuiu com a concepção do estudo, interpretação dos resultados, revisão do texto e aprovação da versão final do manuscrito.

\section{Informações adicionais}

ORCID: Gulnar Azevedo e Silva (0000-0001-87342799); Beatriz Cordeiro Jardim (0000-0002-30752591); Paulo Andrade Lotufo (0000-0002-48568450). 


\section{Referências}

1. Candido DS, Claro IM, Jesus JG, Souza WM, Moreira FRR, Dellicour S, et al. Evolution and epidemic spread of SARS-CoV-2 in Brazil. Science 2020; 369:1255-60.

2. França EB, Ishitani LH, Teixeira RA, Abreu DMX, Corrêa PRL, Marinho F, et al. Óbitos por COVID-19 no Brasil: quantos e quais estamos identificando? Rev Bras Epidemiol 2020; 23:e200053.

3. Azevedo e Silva G, Jardim BC, dos Santos CVB Excesso de mortalidade no Brasil em tempos de COVID-19. Ciênc Saúde Colet 2020; 25:3345-54.

4. Andrade CLT, Pereira CCA, Martins M, Lima SMLL, Portela MC. COVID-19 hospitalizations in Brazil's Unified Health System (SUS). PLoS One 2020; 15:e0243126.

5. Cavalcante JR, Cardoso-Dos-Santos AC, Bremm JM, Lobo AP, Macário EM, Oliveira WK, et al. COVID-19 no Brasil: evolução da epidemia até a semana epidemiológica 20 de 2020. Epidemiol Serv Saúde 2020; 29:e2020376.
6. Consonni D, Coviello E, Buzzoni C, Mensi C. A command to calculate age-standardized rates with efficient interval estimation. Stata J 2012; 12:688-701.

7. Orellana JDY, Cunha GM, Marrero L, Horta BL, Leite IC. Explosão da mortalidade no epicentro amazônico da epidemia de COVID-19. Cad Saúde Pública 2020; 36:e00120020.

8. Szwarcwald CL, Montilla DER, Marques AP, Damacena GN, de Almeida WS, Malta DC. Desigualdades na esperança de vida saudável por Unidades da Federação. Rev Saúde Pública 2017; 51 Suppl 1:7s

9. Vasconcelos AM, Gomes MMF. Transição demográfica: a experiência brasileira. Epidemiol Serv Saúde 2012; 21:539-48.

10. GBD 2016 Brazil Collaborators. Burden of disease in Brazil, 1990-2016: a systematic subnational analysis for the Global Burden of Disease Study 2016. Lancet 2018; 392:760-75.

11. Dal Poz MR, Levcovitz E, Bahia L. Brazil's fight against COVID-19. Am J Public Health 2021; 111:390-1. 
Abstract

The sharp growth in COVID-19 cases and deaths has created a heavy overburden on Brazil's health system, especially in the cities of Manaus (Amazonas State), Rio de Janeiro, and São Paulo. The description of the pandemic's impact has been based on absolute numbers and crude mortality rates, failing to consider the age distribution patterns in different regions of the country. This study aims to compare the crude mortality rates from COVID-19 with age-standardized rates in the state capitals and Federal District. Information on deaths was accessed in the Information System on Influenza Surveillance (SIVEP-Gripe), and the population denominators were based on the estimate provided by the Brazilian Ministry of Health. Calculation of the age-standardized rates used the estimated age structure of the Brazilian population in 2020. The results show that the highest crude rates were in Manaus (253.6/100,000) and Rio de Janeiro (253.2/100,000). Age standardization led to a major increase in the North of Bra$z i l$. The highest age-adjusted rate was in Manaus (412.5/100,000), where 33\% of COVID-19 deaths occurred in individuals under 60 years of age. Mortality in Manaus over 70 years of age was double that of Rio de Janeiro and triple that of São Paulo. The use of age-adjusted mortality rates eliminates interpretative biases, clearly exposing the even greater weight of COVID-19 in the North of Brazil.

COVID-19; Health Status Disparities; Mortality

\section{Resumen}

El crecimiento acentuado de casos y óbitos por COVID-19 ha provocado una gran sobrecarga del sistema de salud en Brasil, en especial en ciudades como Manaus (Estado del Amazonas), Rio de Janeiro y São Paulo. La descripción del impacto de la pandemia se ha basado en números absolutos o tasas de mortalidad brutas, no considerando el patrón de distribución de las franjas etarias en las diferentes regiones del país. Este estudio tiene como objetivo comparar las tasas de mortalidad brutas por COVID-19, con las tasas estandarizadas por edad, en las capitales de los estados brasileños $y$ en el Distrito Federal. Se accedió a la información sobre fallecimientos en el Sistema de Información de Vigilancia de la Gripe (SIVEP-Gripe), y los denominadores poblacionales se basaron en las estimaciones facilitadas por el Ministerio de Salud de Brasil. Para el cálculo de las tasas estandarizadas por edad, se utilizó la estructura etaria de la población de Brasil estimada para 2020. Los resultados muestran que las mayores tasas brutas se produjeron en Manaus $(253,6 / 100.000)$ y en Rio de Janeiro (253,2/100.000). Tras la estandarización por edad, hubo un aumento expresivo de las tasas en la Región Norte. La mayor tasa ajustada fue vista en Manaus (412,5/100.000), donde un $33 \%$ de óbitos por COVID-19 se produjeron entre menores de 60 años. La mortalidad en Manaus por encima de 70 años fue el doble, si se compara con la de Rio de Janeiro, y el triple si se compara con la de São Paulo. La utilización de tasas de mortalidad estandarizadas por edad elimina sesgos interpretativos, exponiendo, de forma significativa, el peso todavía mayor de la COVID-19 en la Región Norte del país.

COVID-19; Desigualdades en la Salud; Mortalidad
Recebido em 19/Fev/2021

Versão final reapresentada em 01/Abr/2021

Aprovado em 07/Mai/2021 